\title{
Uso de dos técnicas alternativas de manejo de conducta: musicoterapia y distracción audiovisual, en el control y manejo de ansiedad en pacientes pediátricos de 5 a 10 años.
}

\section{Resumen}

En la actualidad, se ha demostrado que la ansiedad influye significativamente en la conducta de los niños durante la consulta dental ocasionando un rechazo total del niño a la atención odontológica, optándose por el uso de técnicas no farmacológicas para el control y manejo de la ansiedad, así conseguir un comportamiento receptivo. Objetivo: Comparar dos técnicas no farmacológicas (musicoterapia y distracción audiovisual) en el control de la ansiedad antes, durante y después del tratamiento dental de niños entre 5 y 10 años atendidos en el Centro Universitario de Salud (UPC). Materiales y métodos: Se realizó un estudio cuasi experimental, comparándose los niveles de ansiedad obtenidos con el Test de Dibujos de Venham (1 -8) y la Escala de Ansiedad de Venham (0-5) a un total de 60 pacientes pediátricos bajo las técnicas no farmacológicas musicoterapia y audio visual.
Lorena Alarco-Cadillo ${ }^{1}$,

Leslie Casas Apayco ${ }^{2}$,

Mario Reyes Bossio ${ }^{3}$,

María Cecilia Ramírez Torres ${ }^{4}$,
Resultados: El promedio de puntaje obtenido antes de la consulta dental con el Test de Dibujos de Venham para el grupo control fue de 2, para el grupo musicoterapia fue de 1,4 y de 1,45 para el grupo audio visual; el promedio de la escala de Ansiedad durante la consulta para el grupo control fue de 1,4 y de 0,2 para musicoterapia y audiovisual. Por último, el puntaje después de la consulta dental para el grupo control fue de 1,95, para musicoterapia fue de 0,45 y para el grupo audio visual fue de 0,3 . Sí se encontraron diferencias significativas para la evaluación de la ansiedad con la Escala de Ansiedad de Venham aplicada durante el tratamiento dental entre los tres grupos de estudio $(p=0,001)$. Conclusiones: Se concluye que existe una reducción de los niveles de ansiedad durante y después de emplear ambas técnicas no farmacológicas.

Palabras Clave: musicoterapia, técnicas, niños, ansiedad, tratamiento dental.

1. Alumno de la Carrera de Odontología de la Universidad Peruana de Ciencias Aplicadas, UPC. Lima-Perú.

2. Doctor en Ciencias Odontológicas Aplicadas, Profesor Investigador de la Carrera de Odontología de la Universidad Peruana de Ciencias Aplicadas,

UPC. Lima-Perú.

3. Docente de la Carrera de Psicología de la Universidad Peruana de Ciencias Aplicadas, UPC. Lima-Perú.

4. Especialista en Odontopediatría y Docente de la Carrera de Odontología de la Universidad Peruana de Ciencias Aplicadas, UPC. Lima-Perú. 


\section{Uso de duas técnicas alternativas de manejo da conduta: musicoterapia e distração audiovisual no controle e manejo da ansiedade em pacientes pediátricos de 5 a 10 anos}

\section{Resumo}

Atualmente pode-se adotar por técnicas não farmacológicas para o controle e manipulação dos problemas de ansiedade em crianças, já que afeta significativamente na conduta delas durante o atendimento odontológico, além terá um efeito negativo na saúde oral, já que um elevado nível de ansiedade produz a rejeição total do tratamento dental. Objetivo: Comparar duas técnicas não farmacológicas (musicoterapia e distração audiovisual) no controle da ansiedade antes, durante e após o tratamento dental em crianças de 5 a 10 anos de idade atendidas no Centro Universitário de Saúde (UPC). Materiais e Métodos: Realizou-se um estudo quase experimental e compararam-se os niveles de ansiedade obtidos pelos desenhos de teste de Venham (1 -8) e a escala de ansiedade Venham (0-5) num total de 60 pacientes pediátricos sob técnicas não farmacológicas musicoterapia e audiovisual. Resultados: A média da porcentagem obtido antes da atenção odontológica utilizando os desenhos de teste de Venham para o grupo controle foi 2, para o grupo musicoterapia 1,4 e de 1,45 para o grupo audiovisual; por outro lado a media após da atenção odontológica para o grupo controle foi 1,95, para musicoterapia 0,45 e para o grupo audiovisual 0,3 . A media da escala da Ansiedade durante a consulta para o grupo controle foi de 1,4 e de 0,2 para musicoterapia e audiovisual. Houve diferença significativa entre os três grupos de estudo para avaliação da ansiedade $(p=0,001)$. Conclusões: Os resultados indicam que houve redução dos níveis de ansiedade após empregar ambas as técnicas não farmacológicas na população estudada.

Palavras chaves: musicoterapia, técnicas, crianças, ansiedade, tratamento dental.

Original article

\section{Use of two alternative techniques for the management of behavior: music therapy and audiovisual distraction, for the control and management of anxiety and child's behavior}

\begin{abstract}
It has been shown that anxiety has a significant influence on a child's behavior during a dental appointment and consequently a negative impact on their oral health. It may cause the child to reject their dental treatment. Today, dentists can
\end{abstract}

choose non-pharmacological techniques for the management and control of anxiety to achieve a receptive patient behaviour. Objectives: The aim of this study was to compare two non-pharmacological techniques and for managing anxiety before, during and after dental treatment in 5 to 10 year-old children treated at the universi- 
ty health center. Material and Methods: A quasi experimental study was carried out and the anxiety levels obtained with the Venham Picture Test (1-8) Venham Anxiety Scale (0-5), were applied in 60 pediatric patients in total, using the non-pharmacological techniques: music therapy and audiovisual distraction. Results: The mean score obtained before the dental appointment with the Venham Picture Test was 2 for the control group; 1.4 for the music therapy group and 1.45 for the audiovisual group. The mean score of Venham Anxiety scale during a dental appointment was 1.4 for the control group

\section{Introducción}

En la atención odontológica de pacientes pediátricos es posible encontrar a niños con comportamientos no receptivos producto de la corta edad, temor al tratamiento dental y a la atención odontológica, experiencias previas desagradables e incluso la ansiedad transmitida por los padres. ${ }^{1}$ La ansiedad genera cuadros de estrés durante la consulta odontológica, alterando el comportamiento del niño durante el tratamiento y como consecuencia cancelación de sus consultas programadas, un mayor tiempo de espera entre citas, y un considerable aumento en la complejidad en los procedimientos odontológicos; por ello es de vital importancia el manejo adecuado de la conducta del paciente pediátrico por parte del odontólogo de tal forma que pueda ganarse la confianza del niño y conseguir una mejora paulatina de su comportamiento durante la atención rutinaria. ${ }^{1,2}$

En pacientes que desarrollan ansiedad pre-operatoria estas reacciones son anticipatorias y ocurren de forma independiente a los estímulos externos. En el caso de los niños, el control y manejo de la ansiedad por parte del odontólogo es una parte and 0.2 for the music therapy and audiovisual group. Lastly, the score after dental appointment was 1.95 for the control group, 0.45 for the music therapy group and 0.3 for the audiovisual group. There is a significant difference among the three groups $(p=0,001)$ for anxiety. Conclusions: We conclude that there was a reduction of anxiety levels during and after using both non-pharmacological techniques in the studied population.

Keywords: music therapy, techniques, children, anxiety, dental treatment.

fundamental del tratamiento ya que de esto dependerá que el paciente pueda vencer sus miedos y tener una cita dental tranquila sin sentir ningún tipo de estrés o temor, esto en un futuro permitirá que acuda periódicamente a sus controles y no asociará la visita dental con una experiencia desagradable. ${ }^{2}$

La Academia Americana de Odontopediatría $(\mathrm{AAPD})^{3}$ sugiere que cada paciente debe ser tratado individualmente y con especial cuidado al momento del tratamiento dental, por tal motivo se han implementado diferentes tipos de técnicas de manejo de conducta, las cuales pueden ser agrupadas en: técnicas farmacológicas, las cuales incluyen el uso de medicamentos ansiolíticos para reducir el nivel de ansiedad durante el tratamiento dental y en determinadas situaciones se apoyan en métodos de restricción física pasiva o activa y las técnicas no farmacológicas que incluyen a las técnicas de manejo de conducta tradicional donde encontramos las técnicas comunicativas como decir - mostrar - hacer, modelamiento, manejo de la contingencia y desensibilización, así como las terapias no invasivas para el control y manejo de la ansiedad como musicoterapia y distracción audiovisual. ${ }^{1,2}$ 


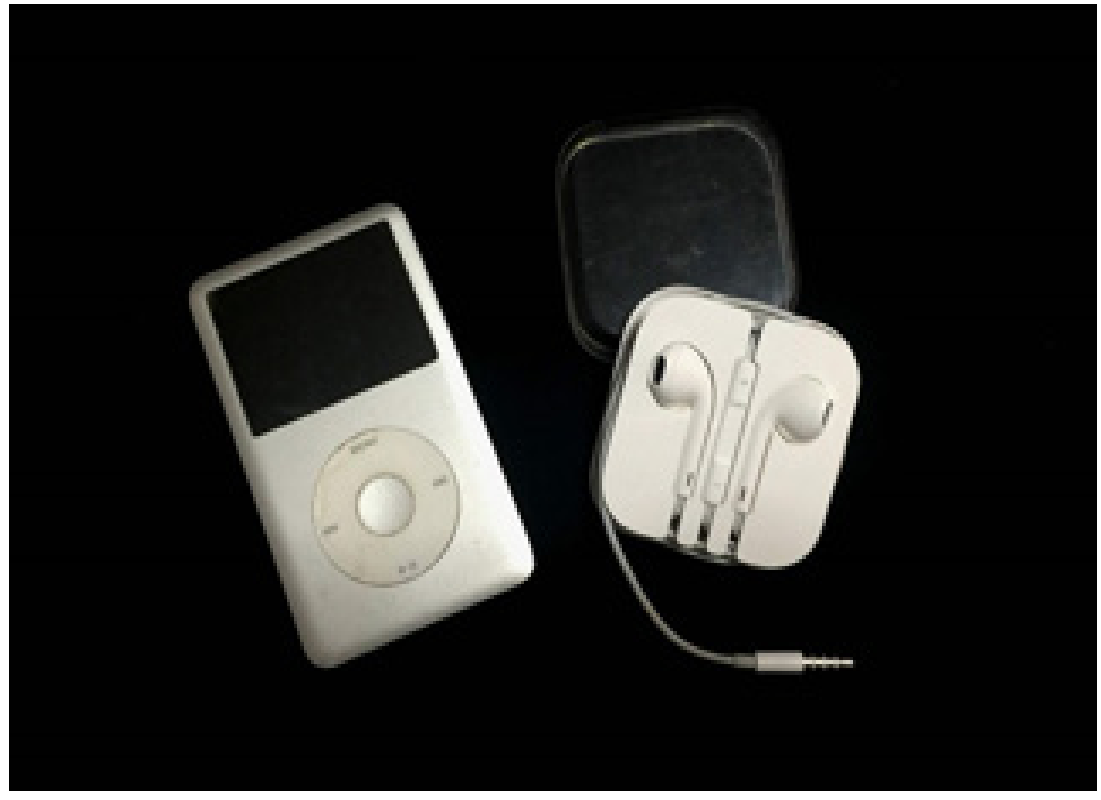

Figura 1. Reproductor MP3 (iPod) y audífono utilizado como distractor musical.

\section{Materiales y métodos}

En este estudio se evalúo una muestra de 60 pacientes de un total de población universal obtenida de la clínica pediátrica del Centro Universitario de Salud, de la Universidad Peruana de Ciencias Aplicadas, Lima - Perú. Los participantes debían cumplir los siguientes criterios de inclusión: niños entre los 5 y 10 años de edad cuyos padres aceptaron la participación de sus hijos el estudio mediante la firma de un consentimiento informado
Estas técnicas alternativas de manejo de conducta como: musicoterapia y distracción audiovisual son efectivas en la modelación conductual en niños no colaboradores o con problemas de comportamiento durante la consulta odontológica con la finalidad de brindar a los padres y al paciente una opción de tratamiento adicional a las técnicas invasivas como restricción física o sedación consciente las cuales conllevan un riesgo para la salud física y emocional del niño. ${ }^{1}$ Además, esto permite tanto a odontopediatras como odontólogos generales mejorar la calidad de sus servicios brindados, una reducción del tiempo de trabajo, disminución de la ansiedad operatoria y una mayor motivación de sus pacientes hacia el cuidado de salud oral. El propósito de este estudio fue comparar dos técnicas alternativas de manejo de conducta (musicoterapia y distracción audiovisual) en el control y manejo de la ansiedad antes, durante y después del tratamiento dental de niños atendidos en el Centro Universitario de Salud de la Universidad Peruana de Ciencias Aplicadas UPC. y la aceptación de los niños mediante la firma de un asentimiento informado. El estudio fue aprobado por el Comité de Ética de la UPC, PI257-15. Se excluyó a los pacientes con comportamiento altamente disruptivo (no colaboradores) y con impedimentos físicos o mentales.

Se conformaron 3 grupos de forma aleatoria, en el primer grupo (Grupo control) los pacientes no recibieron ningún tipo de técnica de musicoterapia o distracción audiovisual, el manejo de conducta se realizó con las técnicas decir - mostrar - hacer y manejo de la contingencia. El segundo grupo (Grupo musicoterapia) utilizó dos reproductores MP3 y dos audífonos los cuales fueron entregados a los pacientes quienes podían escoger dos tipos de música de tipo instrumental previamente cargadas en el equipo (Figura 1). En el tercer grupo (Grupo distracción audiovisual) se entregó a los pacientes un par de lentes de realidad virtual Google Cardboard, con cuatro películas animadas de libre elección, y un par de audífonos (Figura 2). Para realizar la evaluación de la ansiedad pre y post-operatoria se utilizó como instrumento el Test de Dibujos de Venham ${ }^{4}$ 


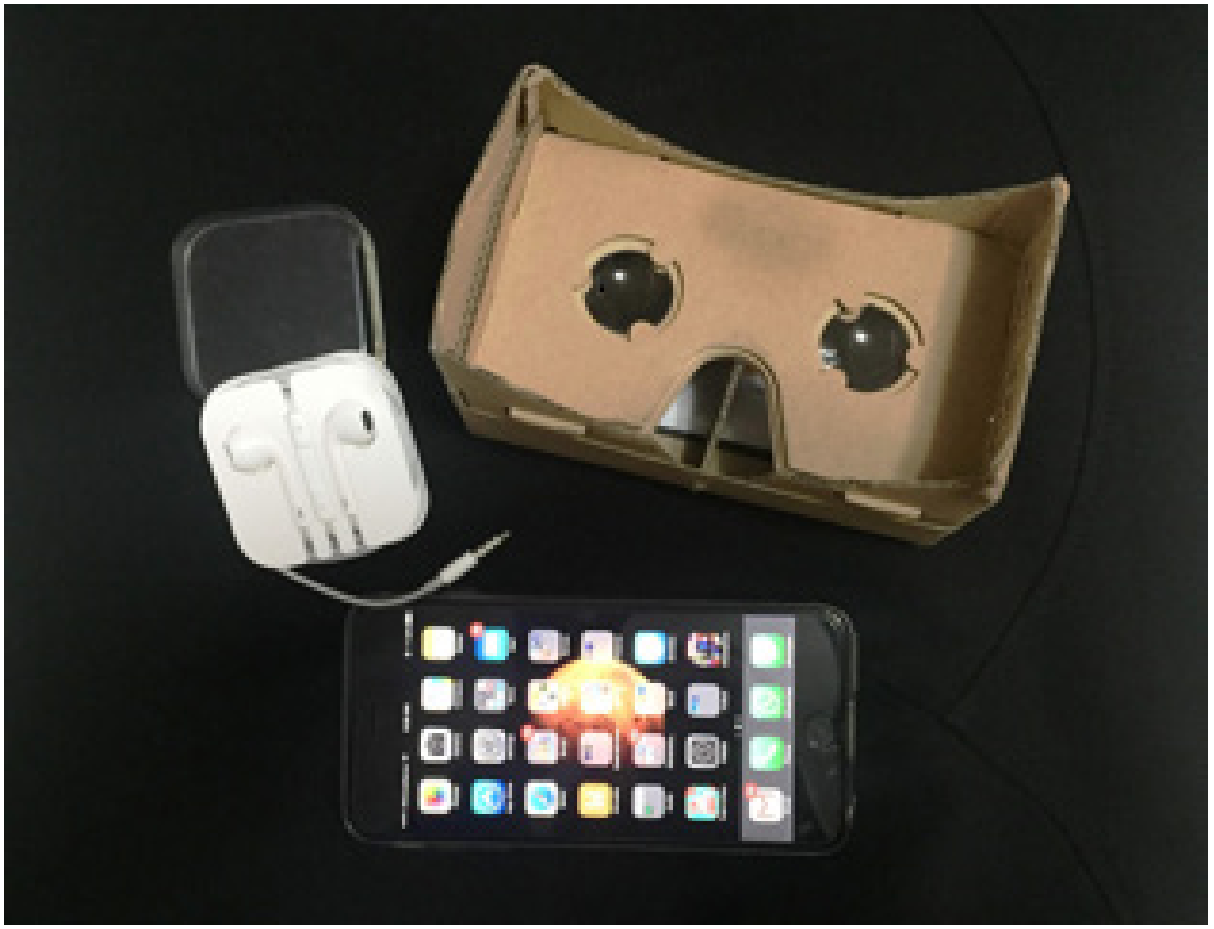

Figura 2. Visor Google Cardboard, celular Smartphone (iPhone) y audífonos utilizados como distractor audiovisual.

y para la evaluación durante el tratamiento dental se utilizó la Escala de Ansiedad de Venham. ${ }^{5}$

La evaluación fue realizada por un solo observador antes, durante y después del procedimiento dental de cada niño. Los niños fueron tratados únicamente bajo procedimientos restauradores (sellantes preventivos o invasivos, restauraciones simples y compuestas) se evaluó a cada paciente de manera individual y objetiva. El tiempo de evaluación para cada paciente no fue mayor a los 60 minutos.

Para la evaluación de la ansiedad pre y post operatoria se utilizó el Test de dibujos de Venham, el cual consta de ocho pares de dibujos, cada par de imágenes representar a un niño calmando, tranquilo o relajado y la otra imagen representa a un niño ansioso, 1lorando, asustado o corriendo. El puntaje está representado por una escala de 1 a 8 , donde los valores de 1 a 4 no representan ansiedad y los valores de 5 a 8 están relacionados con un cuadro de ansiedad
(Figura 3). ${ }^{4}$ Durante el tratamiento dental, se evaluó el comportamiento de cada niño utilizando la Escala de Ansiedad de Venham, la cual está compuesto por 6 categorías, donde 0 indica que el niño es completamente colaborador o sin ansiedad y 5 negativamente colaborador o con mucha ansiedad. ${ }^{5}$

Se evalúo la distribución normal de la muestra por medio del análisis de Shapiro-Wilk y se realizaron medidas de tendencia central y dispersión para la variable ansiedad en los grupos musicoterapia y distracción audiovisual como parte del análisis univariado. Para comparar la ansiedad de los grupos estudiados antes y después del tratamiento se utilizaron las pruebas de Kruskall Wallis y Wilcoxon respectivamente. Los resultados fueron analizados mediante el paquete estadístico Stata ${ }^{\circledR}$ versión $12.0(p<0,05)$.

\section{Resultados}

Al comparar el nivel de ansiedad pre y post - operatorio del tratamiento dental utilizando el Test de Dibujos de Venham, no se encontró diferencias significativas en el grupo control $(p=0,8997)$, a diferencia del grupo musicoterapia $(\mathrm{p}=0,0013)$ y distracción audiovisual $(\mathrm{p}=0,0005)$. (Gráfico 1)

Se encontró que para el grupo control, el promedio de puntaje obtenido al evaluar la ansiedad pre operatoria fue de 2+/- 1,55; el promedio de puntaje 
obtenido durante el tratamiento fue de 1,4 +/- 0,75; mientras que el promedio de puntaje postoperatorio fue de 1,9+/-1,19. Para el grupo musicoterapia, el promedio de puntaje obtenido al evaluar la ansiedad antes del tratamiento (pre - operatoria) fue de 1,4+/-1,18; el promedio de puntaje obtenido durante el tratamiento fue de $0,2+/-0,52$; mientras que el promedio de puntaje después del tratamiento (post - operatorio) fue de 0,45+/-0,75. Finalmente, para el grupo audiovisual, el promedio de puntaje obtenido al evaluar la ansiedad pre - operatoria fue de 1,45 +/- 1,43; el promedio de puntaje obtenido durante el tratamiento fue de $0,2+/-0,52$; mientras que el promedio de puntaje post - operatorio fue de 0,3+/-0,8. (Gráfico 2)

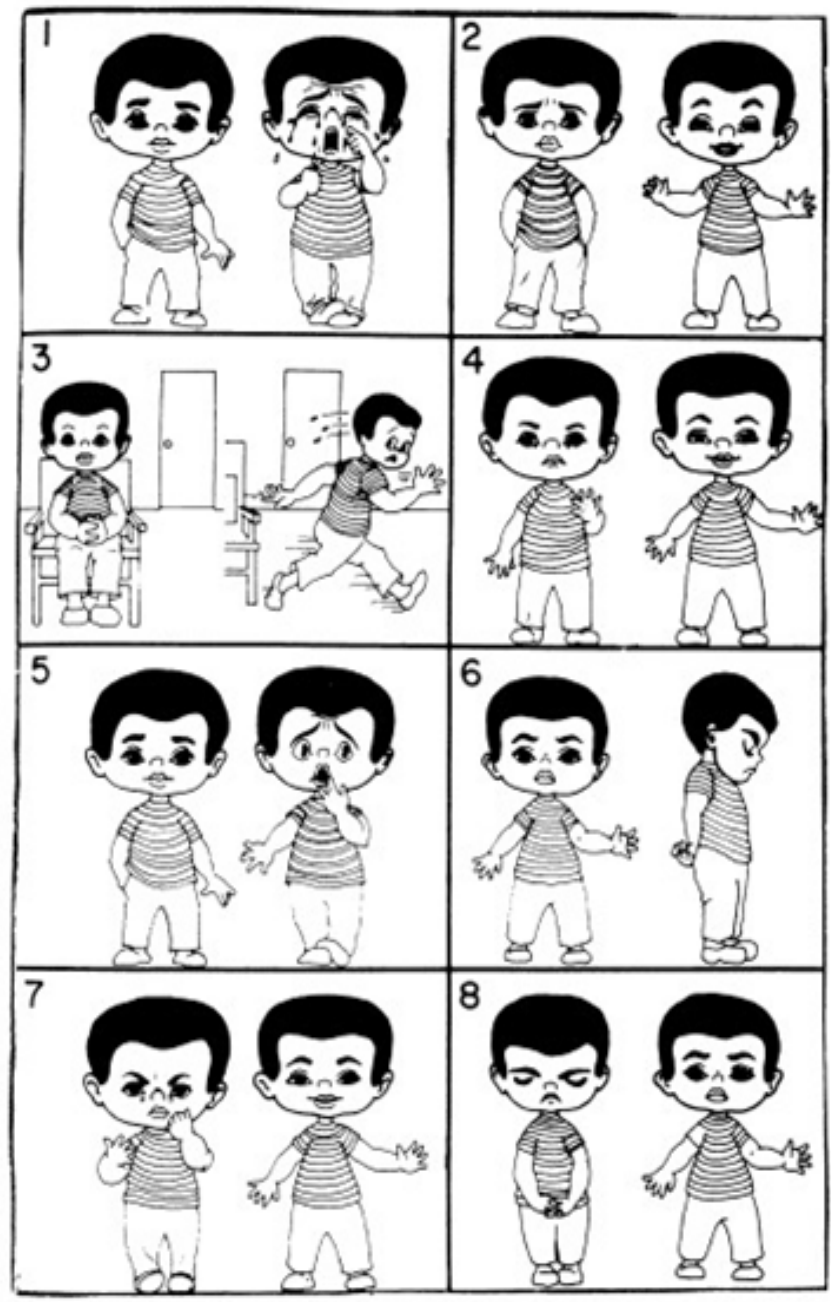

Figura 3. Test de Dibujos de Venham (test pre y post operatorio). Tomado de: Venham L, 1979. ${ }^{3}$

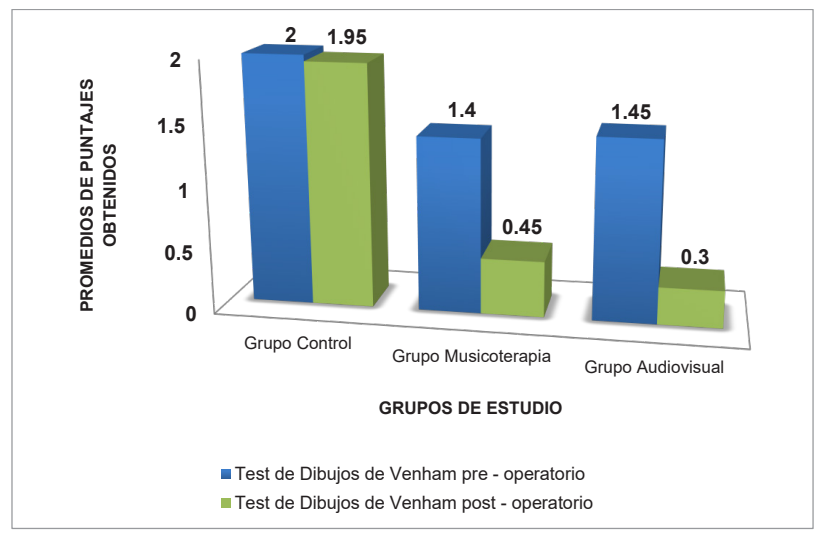

Gráfico 1. Comparación del nivel de ansiedad pre-operatorio y post-operatorio del tratamiento dental de los grupos control, musicoterapia y distracción audiovisual con el Test de Dibujos de Venham.

En la evaluación de la ansiedad pre - operatoria con el Test de Dibujos de Venham se encontró que no hubo diferencias significativas entre los tres grupos de estudio $(\mathrm{p}=0,4962)$. Sin embargo sí se encontraron diferencias significativas para la evaluación de la ansiedad con la Escala de Ansiedad de Venham aplicada durante el tratamiento dental $(p=0,001)$ entre los tres grupos de estudio, asimismo para la evaluación post - operatoria con el Test de Dibujos de Venham $(p=0,0001)$ entre los tres grupos de estudio. (Gráfico 3)

\section{Discusión}

Se calcula que $16 \%$ de los niños en edad escolar presentan cuadros de ansiedad durante los tratamientos dentales y por lo tanto evitan las visitas al odontólogo, lo cual podría mantenerse hasta la adolescencia $^{6}$. Este estudio tuvo como finalidad orientar al odontólogo en el manejo de los pacientes pediátricos utilizando técnicas alternativas para el control de la ansiedad durante la consulta odontológica con el objetivo de generar un cambio de comportamiento, disminuir los niveles de ansiedad durante las citas y conseguir una conducta receptiva en el niño. Diversas investigaciones como 


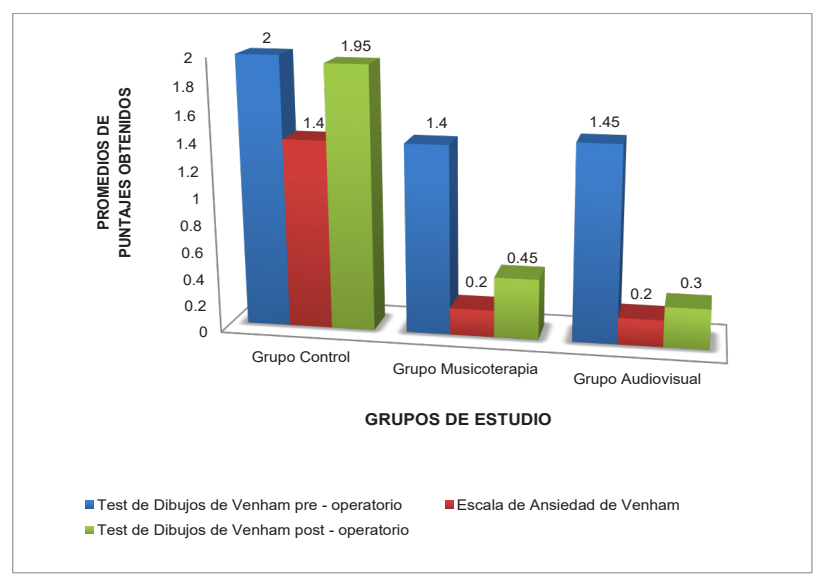

Gráfico 2. Evaluación de la ansiedad para los grupos control, musicoterapia y distracción audiovisual antes, durante y después del tratamiento dental utilizando el Test de Dibujos de Venham y la Escala de Ansiedad de Venham.

las de Naithani y Viswanath ${ }^{7}$ en el 2014 y $\operatorname{Ram}^{8}$ y Col. en el 2010 utilizaron las técnicas de musicoterapia y distracción audiovisual para obtener un mejor control de ansiedad en la consulta dental similares a las usadas en este estudio.

Se optó por elegir pacientes de los primeros ciclos del curso Clínica Pediátrica del Centro Universitario de Salud de la UPC por ser alumnos que realizan únicamente procedimientos restauradores simples y poco extensos. La primera técnica alternativa empleada fue la musicoterapia, la cual

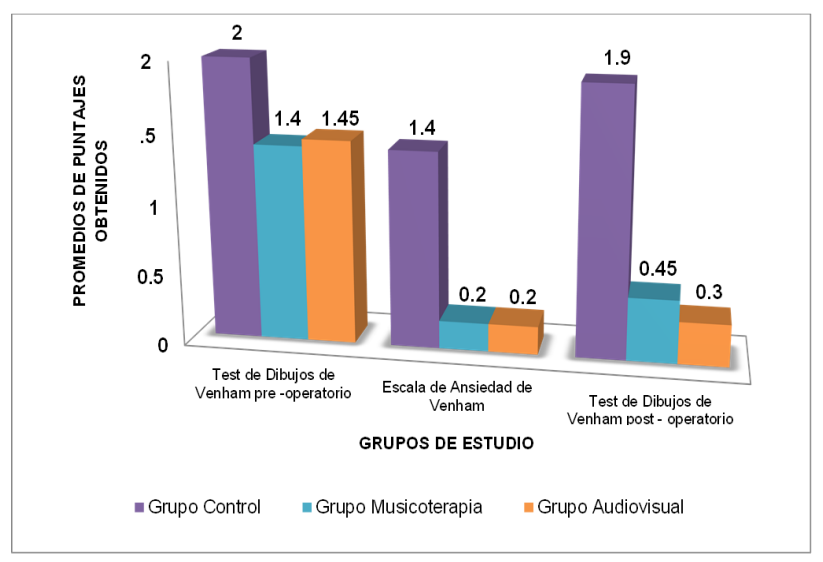

Gráfico 3. Comparación de los grupos control, musicoterapia y audiovisual antes, durante y después del tratamiento con el Test de Dibujos de Venham y la Escala de Ansiedad de Venham. se basa en la música como el elemento clave para conseguir un adecuado manejo del paciente ansioso. Investigaciones realizadas por Marwah y Col. ${ }^{9}$, Divya y Col.,10 Singh y Col. ${ }^{11}$ demostraron que al utilizar esta técnica se pueden conseguir efectos positivos en el ámbito psicológico y fisiológico. La segunda técnica utilizada en este estudio fue la distracción audiovisual, la cual consigue aislar al paciente de manera auditiva y visual, trasladándolo totalmente fuera del entorno odontológico. ${ }^{12}$ En un estudio publicado por Mikala y Col. ${ }^{13}$ en el 2011, se comprobó que los pacientes que utilizaron "video - lentes" presentaron un mejor comportamiento frente a los diferentes tratamientos realizados durante la consulta odontológica.

Los resultados de este estudio se evaluaron de forma cuantitativa y para su desarrollo se utilizó el Test de Dibujos de Venham para evaluar la ansiedad pre - operatoria y post - operatoria. ${ }^{6}$ Las ventajas que posee este test es su fácil manejo, comprensión y rapidez de aplicación, ya que al ser una herramienta que solo contiene imágenes, es accesible a niños a partir de los 4 años de edad. ${ }^{12} \mathrm{Al}$ realizar la comparación de los niveles de ansiedad pre y post - operatoria para el grupo control, el grupo musicoterapia y el grupo distracción audiovisual, se encontró que sí hay disminución de la ansiedad asociada al uso estas técnicas alternativas.

Para el grupo control, los niveles de ansiedad se mantienen con puntajes muy similares al inicio y al final de la consulta odontológica no encontrándose diferencias estadísticamente significativas, en contraste con los grupos de musicoterapia y distracción audiovisual donde sí se observa una considerable disminución entre los niveles de ansiedad pre y post- operatoria de 1,4 a 0,45 para el grupo musicoterapia y de 1,45 a 0,3 para el grupo distracción audiovisual encontrándose diferencias estadísticamente significativas. 
Se encontró en el test pre - operatorio un puntaje de 2 para el grupo control en comparación con los puntajes de 1,4 y 1,45 obtenidos por los grupos de musicoterapia y distracción audiovisual respectivamente no encontrándose diferencias significativas entre los grupos de estudio; del mismo modo en los estudio de Singh D y Col., ${ }^{11}$ Navit S y Col. ${ }^{13} \mathrm{y}$ Aitken JC y Col. ${ }^{14}$ realizado en niños utilizando la musicoterapia como único distractor no se encontraron diferencias significativas en la reducción de la ansiedad para los grupos control y musicoterapia al aplicar este Test antes de iniciar la consulta odontológica.

Para evaluar la ansiedad durante el procedimiento dental, se utilizó la Escala de Ansiedad de Venham $^{5}$, esta escala ha sido una herramienta empleada en múltiples estudios de comportamiento en el ámbito odontopediátrico por la claridad y especificidad de sus respuestas para cada nivel del 1 al 5; y permite al mismo odontólogo investigador evaluar al paciente. Con esta escala el grupo control tuvo un puntaje de 1,4 en comparación con los puntajes de 0,2 obtenidos por los grupos de musicoterapia y distracción audiovisual; esto quiere decir que sí hubo una disminución en los niveles de ansiedad durante el tratamiento. Resultados similares fueron obtenidos en el estudio Naithani y Viswanath ${ }^{7}$ para el grupo distracción audiovisual, donde el puntaje fue de 0,36 indicando una disminución casi total de la ansiedad. Al evaluar la ansiedad post - operatoria el grupo control obtuvo un puntaje de 1.9 en comparación con los puntajes de 0,45 y 0,3 obtenidos por los grupos de musicoterapia y distracción audiovisual respectivamente encontrando diferencias significativas en la reducción de la ansiedad para esta última evaluación; no obstante, los resultados encontrados en el estudio de Aitken JC y Col. ${ }^{14}$ utilizando la musicoterapia como única técnica no se encontraron diferencias significativas entre los grupos control y musicote- rapia al aplicar este Test después de realizar la consulta odontológica.

El presente estudio encontró que ambas técnicas alternativas obtuvieron resultados positivos en el control y manejo de la ansiedad durante y después del tratamiento restaurador, esto proporcionará al odontólogo dos nuevas estrategias a las más empleadas como son: decir - mostrar - hacer, desensibilización, modelamiento y manejo de la contingencia. Estas dos nuevas opciones contribuirán a disminuir el nivel de ansiedad facilitando el manejo de conducta y como consecuencia el odontólogo obtendrá una mejora en el comportamiento del niño, lo cual permitirá reducir el tiempo de consulta odontológica, mejorar la calidad del servicio brindado, mayor aceptación del tratamiento por los padres y conseguir una modificación paulatina de la conducta hacia una actitud positiva frente a la atención odontológica de rutina.

\section{Conclusiones}

Al utilizar técnicas alternativas en el control y manejo de la ansiedad en pacientes pediátricos el odontólogo puede brindar más opciones de tratamiento que no conllevan a un riesgo innecesario en la salud del paciente. Por otro lado, constituyen una opción para aquellos padres que no aceptan las técnicas invasivas o de restricción física como parte de la consulta odontológica.

La distracción audiovisual obtuvo mejores resultados ya que consigue aislar al niño visual y auditivamente del entorno odontológico, reduciendo significativamente los niveles de ansiedad durante y después del tratamiento, permitiendo reducir el tiempo de las citas y consiguiendo una aceptación del tratamiento por parte de los niños. 


\section{Referencias bibliográficas}

1. Quiroz-Torres J, Melgar R. Manejo de conducta no convencional en niños: Hipnosis, musicoterapia, distracción audiovisual: Revisión sistemática. Rev Estomatol Herediana. 2012; 22(2):129-36.

2. Rivera I, Fernández A. Ansiedad y miedos dentales en escolares Hondureños. Rev Latam Psicol. 2005; 37(3): 461-75.

3. American Academy of Pediatric Dentistry (AAPD) [online], Accesado (17 Oct. 2016). Disponible en URL: http://www.aapd.org.

4. Venham L, Gaulin-Kremer E. A Self-report Measure of Situational Anxiety for Young Children. J Clin Pediatr Dent. 1979; 1(2): 94-5.

5. Venham L, Gaulin-Kremer E, Munster E. Interval Rating Scales for Children's Dental Anxiety and Uncooperative Behavior. Pediatr Dent. 1980; 2(3): 198-202.

6. Marcano, A.A., Figueredo, A.M., Orozco, G. Evaluación de la ansiedad y miedo en niños escolares en la consulta odontopediátrica. Rev Odontoped Latinoam 2012; 2(2). Obtenible en: http://www.revistaodontopediatria.org/ediciones/2012/2/ art-7/. Consultado el: 17/10/2016.

7. Naithani M, Viswanath D. Child's Dental Anxiety: Management by audio and audio-visual Distraction Technique - a comparative study. Univ Res J Dent. 2014; 4(2): 1 01-7.

8. Ram D, Shapira J, Holan G, Magora F, Cohen S, Davidovich E. Audiovisual video eyeglass distraction during dental treatment in children. Quintessence Int. 2010; 41:673-9.

9. Marwah N, Prabhakar A, Raju O. Music distraction--its efficacy in management of anxious pediatric dental patients. J Indian Soc Pedod Prev Dent. 2005: 168-70.

10. Divya S, Firoza S, JN J, entre otros. Stress Reduction through Audio Distraction in Anxious Pediatric Dental Patients: An Adjunctive Clinical Study. J Clin Pediatr Dent. 2014; 7(3): 149-152.

11. Singh D, Samadi F, Jaiswal J, Tripathi AM. Stress Reduction through Audio Distraction in Anxious Pediatric Dental Patients: An Adjunctive Clinical Study. Int J Clin Pediatr Dent. 2014 Sep-Dec;7(3):149-52.

12. Magora F, Cohen S, Ram D. Audiovisual Iatrosedation with Video Eyeglasses Distraction Method in Pediatric Dentistry: Case History. J Int Dent Med Res. 2010; 3(3): 133-6.

13. Mikala A, Howard M, Wallace D, Allen K. Use of Video Eyewear to Manage Distress in Children During Restorative Dental Treatment. Pediatr Dent. 2011 ; 34(5): 1 -6.

13. Navit S, Johri N, Khan S, Singh R, entre otros. Effectiveness and Comparison of Various Audio Distraction Aids in Management of Anxious Dental Paediatric Patients. J Clin Diagn Res. 2015; 9(12):5-9.

14. Aitken JC, Wilson S, Coury D, Moursi AM. The effect of music distraction on pain, anxiety and behavior in pediatric dental patients. Pediatr Dent. 2002; 24(2): 114-8.

Recibido: $15 / 08 / 2016$

Aceptado: 04/11/2016

Autor de Correspondencia:

Leslie Casas Apayco, Correo electrónico: leslie.casas@upc.pe

Carrera de Odontología, Universidad Peruana de Ciencias Aplicadas

Dirección: Alameda San Marcos cuadra 2 s/n, Chorrillos, Lima-Perú.

Número de teléfono (511) 313-3333 